\title{
Um Mapeamento Sistemático da Literatura sobre Plugins de Gamificação para o LMS Moodle
}

\author{
Nechelley Alves, Heitor A. X. Costa, Paulo Afonso Parreira Júnior \\ Departamento de Ciência da Computação - Universidade Federal de Lavras \\ Lavras - MG - Brasil \\ mrnechelley@gmail.com, \{heitor, pauloa\}@ufla.br
}

\begin{abstract}
In an LMS such as Moodle, engaging students is a crucial point in learning so that students stay on course and have a desire to learn more. The use of gamification in Moodle can improve students' motivation and engagement by making solving activities and readings more fun. In this work, a Systematic Literature Mapping is presented, in which studies were identified, cataloged and classified whose focus is the application of gamification in Moodle plugins. The goal is to provide an overview on the subject, as well as identify research gaps to be attacked and reveal the main practices adopted by these plugins.
\end{abstract}

Resumo. Em um LMS como o Moodle, o engajamento dos estudantes é um ponto crucial no aprendizado, para que os estudante se mantenham no curso e tenham desejo em aprender mais. O uso de gamificação no Moodle pode aprimorar a motivação e o engajamento dos estudantes, ao tornar a resolução de atividades e leituras mais divertidas. Neste trabalho, é apresentado um Mapeamento Sistemático da Literatura, no qual foram identificados, catalogados e classificados estudos cujo foco é a aplicação de gamificação em plugins do Moodle. O objetivo é fornecer uma visão geral sobre o assunto, bem como identificar gaps de pesquisa a serem atacados e revelar as principais práticas adotadas por estes plugins.

\section{Introdução}

Para suprir uma nova demanda de ensino a distância, dentre as soluções encontradas, estão os Learning Management Systems (LMS) ${ }^{1}$ [Ortiz; Green, 2019]. LMS podem ser definidos como sistemas que automatizam a administração de eventos educacionais, gerenciando o acesso dos usuários registrados, os catálogos de cursos, os dados dos estudantes e fornecendo relatórios administrativos [Paulsen, 2003].

Dentre os LMS existentes na literatura, um dos que possuem maior destaque é o Moodle, devido a sua alta flexibilidade, facilidade de configuração e disponibilização de conteúdos, quantidade de recursos e atividades, entre outros. Atualmente, o Moodle é utilizado por mais de 109 mil instituições, provendo suporte a mais de 18 milhões de cursos e a mais de 155 milhões de alunos em 230 países (Moodle, 2019). Segundo o website oficial do Moodle (2019), o Brasil ocupa a quarta posição de países que mais utilizam a plataforma;

\footnotetext{
${ }^{1}$ Em português, os LMS são, geralmente, nomeados como Ambientes Virtuais de Aprendizagem - AVA.
} 
sendo assim, este LMS se tornou objeto de estudo deste artigo, devido a sua relevância entre os LMS e sua forte participação no cenário brasileiro.

Gamificação, de acordo com Kiryakova, Angelova e Yordanova (2014) apud Kapp (2012), pode ser definida como "usar mecânicas baseadas em jogos, estética e pensamento de jogo para engajar pessoas, motivar ações, promover o aprendizado e solucionar problemas". Como exemplo de gamificação, pode-se considerar desenvolvedores em uma empresa de software que ganham pontos por tarefa concluída e podem trocá-los por prêmios ao final do mês.

A gamificação tem sido explorada no contexto dos LMS, como o Moodle, por meio do uso de plugins (ferramentas que permitem adicionar novas características e funcionalidades ao Moodle, como novas atividades, tipos de quiz, integrações com outros sistemas, entre outros) para essas plataformas. Uma das vantagens de se aplicar gamificação no contexto dos LMS é que eles já possuem ferramentas automáticas, as quais rastreiam as atividades dos alunos, seu progresso e seus resultado, além de fornecerem um ambiente cooperativo e colaborativo por meio do qual, com o uso de estratégias de gamificação, é possível aumentar o engajamento dos alunos em fóruns, blogs e discussões (Kiryakova et al., 2014).

Contudo, atualmente há escassez de trabalhos que se proponham a fazer uma sistematização do conhecimento, a respeito dos plugins existentes para gamificação no LMS Moodle. Aliado a isso, tem-se que, atualmente, o LMS Moodle conta com mais de 1600 plugins disponíveis em sua plataforma de hospedagem e disponibilização de plugins (https://moodle.org/plugins/), dificultando o trabalho de quem deseja encontrar um plugin adequado às suas necessidades. Um trabalho que se assemelha ao propósito deste artigo é o proposto por Dicheva et al. (2015), no qual os autores fizeram um Mapeamento Sistemático da Literatura (MSL) sobre gamificação na educação. Contudo, o trabalho trata o assunto de forma geral, i.e., não focada no Moodle. Entende-se que um estudo mais específico sobre o Moodle é necessário, pois pode favorecer professores que queiram utilizar gamificação em suas disciplinas, bem como desenvolvedores que desejem criar plugins de gamificação para esta plataforma. No caso dos desenvolvedores, a disseminação inadequada do conhecimento sobre este assunto pode acabar levando-os a "reinventarem a roda".

Neste contexto, o objetivo deste trabalho é identificar, classificar e catalogar os principais plugins de gamificação para o LMS Moodle. Para isto, será realizado um MSL, que é um método para identificar, avaliar e interpretar o conhecimento acerca de uma área, com o objetivo de resumir e centralizar este conhecimento (Kitchenham; Charters, 2007). O restante deste artigo está organizado da seguinte forma. Na Seção 2, é explicado sobre como é feito um MSL e são definidas as características do MSL deste trabalho, como as questões de pesquisa e os critérios de inclusão/exclusão. Na Seção 3, os detalhes de como ocorreu o MSL são apresentados e seus resultados são discutidos. Na Seção 4, é apresentada a conclusão do trabalho, destacando os principais pontos encontrados e possíveis projetos futuros.

\section{Planejamento do MSL}

Mapeamento Sistemático da Literatura (MSL) é um procedimento que visa a identificar, avaliar e interpretar o conhecimento relativo a uma área ou tema específico (Kitchenham; Charters, 2007). Um MSL geralmente possui os seguintes passos (Petersen et al., 2008): (i) definição da pesquisa, no qual é definido o tema central do MSL, as questões que serão respondidas, as fontes de busca e os critérios de inclusão/exclusão de estudos; (ii) busca por 
estudos e triagem dos mesmos, com base nos critérios selecionados; e (iii) extração de informação contida nos estudos selecionados e apresentação dos resultados.

\subsection{Questões de Pesquisa}

Durante a condução de um MSL, um passo fundamental é a formulação das questões de pesquisa, que ditam o objetivo do mapeamento e o que será extraído de informação dos estudos considerados como relevantes. Sendo assim, seguem as questões de pesquisa formuladas para este trabalho.

Q1: Quais elementos do conceito de gamificação tem sido aplicados aos plugins do LMS Moodle? Justificativa: descobrir o que, referente à gamificação, já foi aplicado para entender o que é mais comum de ser utilizado e o que ainda pode ser melhor abordado.

Q2: Quais elementos didáticos (atividades, fóruns, quizzes, entre outros) do Moodle têm sido mais adotados nos plugins de gamificação? Justificativa: entender como os elementos didáticos do Moodle contribuem para o uso de gamificação nos plugins e qual a relação existente entre gamificação e o Moodle.

Q3: Como tem sido avaliados os plugins existentes, por parte de seus usuários? Justificativa: entender quais plugins são mais bem avaliados pelos usuários pode favorecer o processo de tomada de decisão dos interessados em utilizar gamificação em seus ambientes de trabalho.

\subsection{String e fontes de busca}

Como fonte de busca, foi utilizado o repositório de plugins oficial do Moodle (https://moodle.org/plugins/). A busca foi realizada no dia 01/05/2019, utilizando-se o mecanismo de pesquisa disponibilizado pela plataforma e a string: "gamification $O R$ gamefication".

Este repositório foi utilizado, pois ele contém os plugins oficiais e autorizados pela equipe de desenvolvimento do Moodle. Todos os plugins disponibilizados neste canal passam por testes automáticos e manuais e precisam da aprovação dos moderadores do Moodle para serem publicados.

\subsection{Critérios de inclusão/exclusão}

É bem comum que, durante a busca por estudos, seja encontrada uma quantidade de documentos muito grande e, dentre eles, apenas alguns estejam realmente relacionados ao tema definido no MSL. Para contornar esta situação, são criados critérios de inclusão/exclusão, os quais servem para definir quais materiais são relevantes para o MSL em questão. Os critérios de inclusão definidos neste trabalho são: (i) o texto de descrição do plugin deve estar escrito em inglês ou português; (ii) o plugin não é uma versão duplicada ou mais antiga de outro plugin; (iii) o plugin diz respeito à gamificação. Como critério de exclusão, tem-se que: se um ou mais critérios de inclusão não for atendido, o plugin deve ser excluído da análise deste MSL.

\section{Execução do MSL, Resultados e Discussão}

Nesta seção, são apresentados os resultados obtidos em cada etapa do MSL realizado, tais como o número de plugins encontrados, aceitos e rejeitados, em cada passo, entre outros. 
VIII Congresso Brasileiro de Informática na Educação (CBIE 2019)

Anais do XXV Workshop de Informática na Escola (WIE 2019)

\subsection{Etapas da execução do MSL}

A seguir estão descritas as etapas realizadas durante o MSL, bem como os resultados obtidos em cada uma delas.

Etapa 1: consistiu em realizar buscas na fonte de pesquisa selecionada, utilizando a string desenvolvida. Feito isso, o repositório de plugins do Moodle retornou 18 plugins relacionados a gamificação.

Etapa 2: a partir da etapa 1, foi feita uma filtragem dos resultados. Para isso, foi lido todo o texto de descrição de cada um dos 18 plugins, aplicando os critérios de inclusão/exclusão. Ao final desta etapa, chegou-se ao número de 17 plugins aceitos e 1 plugin rejeitado, pois o mesmo focava apenas em facilitar a exibição de itens do inventário, não agregando nenhum elemento de gamificação.

\subsection{Resultados e Discussão}

Na Tabela 1, estão os plugins aceitos, após a execução de todas as etapas do MSL. A primeira coluna da tabela apresenta um código de identificação que será utilizado ao longo deste artigo; a segunda coluna, por sua vez, apresenta o nome do plugin; por fim, a terceira coluna apresenta o link onde o plugin está hospedado, sendo que o último acesso a estes links foi realizado em Maio/2019.

O plugin [1] permite atribuir pontos a estudantes automaticamente, cria um sistema de nível por estudante, exibe um ranking dos estudantes e restringe áreas do curso ao nível em que o estudante se encontra. O plugin [2] adiciona pontos aos estudantes, conforme certas atividades vão sendo concluídas e também exibe um ranking de pontuação dos estudantes. $O$ plugin [3] adiciona um inventário de itens aos estudantes e permite aos professores disponibilizar itens a serem ganhos quando atividades são concluídas.

O plugin [4] é dependente do plugin [3]. O plugin [4] permite que professores usem os itens do inventário, provenientes do plugin [3], como restrição para acesso a determinadas áreas do curso. O plugin [5] permite que estudantes reajam a atividades, selecionando emojis que demonstrem sua reação à atividade e ao seu nível de dificuldade. O plugin [6] permite que professores criem testes e exibe o resultado dos estudantes nesses testes em um ranking.

O plugin [7] permite que os estudantes tenham créditos em seu perfil que podem ser usados para comprar cursos; estes créditos são adicionados por meio de pagamento externo à plataforma Moodle. Como elemento de gamificação, além das funcionalidades já informadas, o plugin disponibiliza um sistema de pontuação e permite que estudantes comprem cursos usando estas pontuações, sem a necessidade do depósito de dinheiro. O plugin [8] permite que os professores criem quizzes e outros jogos de texto (jogos em que as ações do estudante se limitam a escrever textos), como um jogo de forca ou um jogo onde o estudante precisa digitar o nome daquilo que vê nas imagens. O plugin [9] permite que os professores criem quizzes com imagens e incentivos visuais ao estudante, oferecendo um visual de jogo.

O plugin [10] é dependente do [1]. O plugin [10] permite ao professor bloquear certas áreas do curso usando pontuações e níveis como parâmetro, sendo estas pontuações e níveis provenientes do plugin [1]. O plugin [11] permite que o professor crie insígnias e as entregue a seus alunos. O plugin [12] permite criar insígnias, porém não possui sistema para entregá-las, sendo necessário outro plugin que faça isso. 
VIII Congresso Brasileiro de Informática na Educação (CBIE 2019)

Anais do XXV Workshop de Informática na Escola (WIE 2019)

Tabela 1. Plugins analisados para responder às questões de pesquisa do MSL.

\begin{tabular}{|c|c|c|}
\hline \# & Nome & Link \\
\hline 1 & Level up! - Gamification & https://moodle.org/plugins/block_xp \\
\hline 2 & Ranking block & https://moodle.org/plugins/block_ranking \\
\hline 3 & Stash & https://moodle.org/plugins/block_stash \\
\hline 4 & Stash availability & https://moodle.org/plugins/availability_stash \\
\hline 5 & Point of view & https://moodle.org/plugins/block_point_view \\
\hline 6 & UNEDTrivial (spaced quizzes) & https://moodle.org/plugins/mod_unedtrivial \\
\hline 7 & Course credit enrolment & https://moodle.org/plugins/enrol_credit \\
\hline 8 & Game & https://moodle.org/plugins/mod_game \\
\hline 9 & Quizventure & https://moodle.org/plugins/mod_quizgame \\
\hline 10 & Level up! Availability & https://moodle.org/plugins/availability_xp \\
\hline 11 & Stamp collection & https://moodle.org/plugins/mod_stampcoll \\
\hline 12 & Badge Pool & https://moodle.org/plugins/local_bs_badge_pool \\
\hline 13 & Badge Ladder & https://moodle.org/plugins/local_bs_badge_ladder \\
\hline 14 & Recent badges & https://moodle.org/plugins/block_bs_recent_badges \\
\hline 15 & Exabis Games & https://moodle.org/plugins/mod_exagames \\
\hline 16 & Open Badge Factory displayer block & https://moodle.org/plugins/block_obf_displayer \\
\hline 17 & Pedagogic Achievements & https://moodle.org/plugins/block_achievement \\
\hline
\end{tabular}

O plugin [13] disponibiliza dois tipos de ranking; um deles mostra as insígnias existentes e quantos estudantes a conquistaram e o outro exibe todos os estudantes, ordenados pela quantidade de insígnias adquiridas, sendo que o plugin em si não cria insígnias apenas as exibe em ranking. O plugin [14] disponibiliza um ranking, informando quais foram as últimas insígnias adquiridas, sendo que, como o plugin [13], este não cria insígnias apenas as exibe em ranking. O plugin [15] permite a criação de quizzes animados, semelhante ao plugin [9].

O plugin [16] permite que insígnias sejam exibidas, por exemplo, no perfil do estudante. O plugin [17] entrega insígnias aos estudantes conforme eles executem certas ações no cursos, sendo estas ações já predefinidas pelo plugin.

\subsubsection{Resposta à $Q P 1$}

Esta questão buscou identificar quais elementos de jogos têm sido aplicados nos plugins do Moodle. A Tabela 2 apresenta os elementos identificados a partir dos plugins analisados, bem como o código dos plugins que contemplam tais elementos. 
VIII Congresso Brasileiro de Informática na Educação (CBIE 2019)

Anais do XXV Workshop de Informática na Escola (WIE 2019)

De acordo com a Tabela 3, nota-se que o uso de rankings e insígnias, ambos com 5 plugins cada, são os mais utilizados nos plugins. Um motivo para isto pode ser a facilidade de implementação destes elementos e também por eles serem elementos bem recorrentes e conhecidos em jogos.

Tabela 2. Elementos de gamificação elencados a partir dos plugins analisados.

\begin{tabular}{|l|l|}
\hline \multicolumn{1}{|c|}{ Elementos de jogos e sua descrição } & Plugins \\
\hline $\begin{array}{l}\text { Ranking: apresentação dos estudantes ou insígnias na tela, usando algum critério de } \\
\text { ordenação. }\end{array}$ & {$[1][2][6][13][14]$} \\
\hline $\begin{array}{l}\text { Nível: valor numérico caracterizado de acordo com a pontuação do estudante, } \\
\text { normalmente quanto maior a pontuação maior será o nível do estudante. }\end{array}$ & {$[1][10]$} \\
\hline $\begin{array}{l}\text { Pontuação: representa de forma quantitativa as tarefas/objetivos concluídos pelo } \\
\text { estudante, normalmente inicia em zero e aumenta a cada tarefa/objetivo concluído. }\end{array}$ & {$[1][2][7][10]$} \\
\hline $\begin{array}{l}\text { Insígnias: utilizado como forma de recompensar o estudante conforme ele avance no } \\
\text { curso, ou execute ações importantes para si ou para o curso. }\end{array}$ & {$[11][12][13][14][16]$} \\
\hline $\begin{array}{l}\text { Itens: elementos que podem ser adquiridos conforme objetivos são concluídos e } \\
\text { podem ser usados para acessar áreas restritas do curso. }\end{array}$ & {$[3][4]$} \\
\hline Reação: elemento onde as atividades recebem características dadas pelos estudantes. & {$[5]$} \\
\hline $\begin{array}{l}\text { Quiz: questionário com perguntas que devem ser respondidas, tendo este que } \\
\text { apresentar formas de engajamento que se assemelham a jogos, para que o estudante } \\
\text { se sinta jogando um quiz com incentivos visuais e não apenas respondendo } \\
\text { perguntas. }\end{array}$ & {$[6][8][9][15]$} \\
\hline $\begin{array}{l}\text { Restrição de área: restrição de certa área do curso até que certo desafio seja } \\
\text { concluído. }\end{array}$ & {$[1][4][7][10]$} \\
\hline
\end{tabular}

$\mathrm{Na}$ Tabela 3, é possível visualizar quais elementos de gamificação tendem a aparecer juntos, nos plugins desenvolvidos. Com isso, é possível averiguar que o mais comum é que sejam usadas pontuações para restringir áreas do curso, o que faz sentido, dado que a pontuação pode ser considerada como uma medida de progresso do estudante no curso. Assim para se ter acesso às atividades mais difíceis, é necessário executar, primeiramente, as mais fáceis, ganhando pontos e experiências com elas. Outro ponto a se ressaltar é a popularidade do uso de sistemas de pontuações e insígnias para formação de rankings de estudantes.

\subsubsection{Resposta à $Q P 2$}

Esta questão buscou entender como os elementos didáticos do Moodle são usados nos plugins. A Tabela 4 apresenta a relação entre os elementos didáticos do Moodle e os plugins, destacando que o uso de quizzes e a restrição de áreas do curso, ambas com 4 plugins, são as mais utilizadas, enquanto que o uso do fórum é menos abordado, tendo apenas 1 plugin. Um motivo para isto deve-se ao fato que os quizzes possuem função dupla, ao ensinar o estudante com seus erros e ao mesmo tempo fornecer feedbacks ao professores sobre o quanto os estudantes têm acertado as questões. Enquanto que o fórum, por ser um ambiente mais social depende de elementos de gamificação que sejam mais subjetivos, o que teria ocasionado que menos plugins o abordassem. 
VIII Congresso Brasileiro de Informática na Educação (CBIE 2019)

Anais do XXV Workshop de Informática na Escola (WIE 2019)

Tabela 3. Elementos de gamificação que aparecem juntos nos plugins analisados.

\begin{tabular}{|l|l|l|l|l|l|l|l|l|}
\hline & \multicolumn{1}{|c|}{ A } & B & C & D & E & F & G & H \\
\hline [A] Ranking & & & & & & & & \\
\hline$[$ B] Nível & {$[1]$} & & & & & & & \\
\hline$[C]$ Pontuação & {$[1][2]$} & {$[1][10]$} & & & & & & \\
\hline$[$ D] Insígnias & {$[13][14]$} & & & & & & & \\
\hline$[$ E] Itens & & & & & & & & \\
\hline [F] Reação & & & & & & & & \\
\hline [G] Quiz & {$[6]$} & & & & & & & \\
\hline [H] Restrição de área & {$[1]$} & {$[1][10]$} & {$[1][7][10]$} & & {$[4]$} & & & \\
\hline
\end{tabular}

Tabela 4. Elementos didáticos utilizados pelos plugins analisados.

\begin{tabular}{|l|l|}
\hline \multicolumn{1}{|c|}{ Elementos didático e sua descrição } & \multicolumn{1}{|c|}{ Plugins } \\
\hline $\begin{array}{l}\text { Fórum: ambiente no qual os estudantes e professores podem se comunicar por meio de } \\
\text { mensagens de texto. }\end{array}$ & {$[5]$} \\
\hline $\begin{array}{l}\text { Atividades: tarefas que os estudantes podem/devem executar no ambiente ou enviar provas } \\
\text { de que foi concluída. }\end{array}$ & {$[1][2][17]$} \\
\hline Quiz: questionário com perguntas que devem ser respondidas. & {$[6][8][9][15]$} \\
\hline Restrição de área: restrição de certa área do curso até que certo desafio seja concluído. & {$[1][4][7][10]$} \\
\hline
\end{tabular}

\subsubsection{Resposta à $Q P 3$}

Com esta questão, visou-se observar a satisfação e a frequência de uso dos plugins de gamificação no Moodle, a fim de atestar se realmente os plugins têm sido utilizados e se os ganhos no engajamento dos estudantes foram observados por seus utilizadores. Sendo assim, a Tabela 5 apresenta, para cada plugin analisado, quantos sites do Moodle o utilizam, bem como a quantidade de downloads e fãs (todas estas informações podem ser obtidas a partir do repositório de plugins do Moodle).

Com base na Tabela 5, é visível a disparidade entre os plugins [1] e [8], em relação aos demais, em qualquer um dos parâmetros analisados. Seus altos valores se devem, provavelmente, ao fato de o plugin [1] ter um conjunto de elementos de gamificação bem completo, com pontuações, rankings e desbloqueio de áreas, e também por sua compatibilidade com várias versões do Moodle, tendo versões do plugin compatíveis desde a versão 2.7 do Moodle até a versão 3.7 (a versão mais atual do Moodle, em Julho/2019, é a 3.7). Ainda sobre o plugin [1], outro ponto que pode ter influenciado sua popularidade é o fato de outros plugins dependerem diretamente dele ou de alguma funcionalidade que ele fornece, tornando-o atrativo até para usuários que desejem outro plugin.

Já sobre o plugin [8], sua popularidade se deve provavelmente a sua compatibilidade com versões do Moodle de 2.0 à 3.7, além de seu diferencial de fornecer diferentes jogos, 
VIII Congresso Brasileiro de Informática na Educação (CBIE 2019)

Anais do XXV Workshop de Informática na Escola (WIE 2019)

além de quizzes, o tornando mais interessante que outros plugins que oferecem apenas a criação de quizzes como os plugins [9] e [15].

Tabela 5. Informações sobre a utilização dos plugins analisados.

\begin{tabular}{|c|c|c|c|c|}
\hline Plugin & $\begin{array}{l}\text { Quantidade de } \\
\text { instalações }\end{array}$ & $\begin{array}{l}\text { Quantidade de } \\
\text { downloads }\end{array}$ & Quantidade de fãs & $\begin{array}{l}\text { Versões do Moodle } \\
\text { Compatíveis }\end{array}$ \\
\hline [8] & 4995 & 5000 & 151 & 2.0à 3.7 \\
\hline [1] & 3759 & 4000 & 317 & 3.1 à 3.7 \\
\hline [9] & 963 & 438 & 77 & 3.5 à 3.7 \\
\hline$[10]$ & 927 & 377 & 54 & 2.7 à 3.7 \\
\hline [2] & 1175 & 352 & 80 & 3.2 à 3.6 \\
\hline [3] & 514 & 314 & 52 & 3.1 à 3.6 \\
\hline [5] & 108 & 195 & 23 & 3.3 à 3.6 \\
\hline [4] & 304 & 169 & 15 & 2.9 à 3.6 \\
\hline [11] & 302 & 95 & 35 & 3.3 à 3.6 \\
\hline [12] & 139 & 78 & 13 & 3.5 à 3.6 \\
\hline [13] & 119 & 77 & 17 & 3.5 à 3.6 \\
\hline [15] & 45 & 64 & 26 & 2.0 à 2.5 \\
\hline [14] & 71 & 50 & 7 & 3.5 à 3.6 \\
\hline [7] & 8 & 47 & 3 & 3.2 à 3.6 \\
\hline [16] & 33 & 38 & 12 & 3.5 \\
\hline [6] & 14 & 21 & 4 & 3.0 a 3.1 \\
\hline [17] & 12 & 8 & 9 & 2.0 a 2.3 \\
\hline
\end{tabular}

Sobre o plugin [7], que aparece como o menos popular dos analisados, um motivo que pode ter levado a isso é sua criação recente (fevereiro de 2019). Outro ponto é o foco específico do plugin em vender cursos, tendo o uso de pontuação como funcionalidade secundária do plugin, possivelmente afastando usuários que não estão interessantes na comercialização de cursos.

\section{Considerações finais}

A partir das respostas obtidas neste MSL, é possível inferir que já existem plugins que implementam a gamificação no Moodle, porém ainda são pouco utilizados. Se compararmos os 4995 sites que utilizam o plugin [8], isso ainda é menos da metade das mais de 10 mil instâncias que utilizam o plugin mais popular do Moodle (plugin do tema Adaptable ${ }^{2}$ ). Além

${ }^{2}$ https://moodle.org/plugins/theme_adaptable 
disso, existem plugins que abordam diferentes elementos de gamificação, mas poderiam estar juntos em um único plugin, tornando-se um produto mais robusto e que agregue mais valor ao site.

Com base nos plugins, é possível verificar que a maioria tende a seguir a gamificação pelo modelo de premiação (com pontos, itens ou insígnias) conforme as atividades (objetivos, tarefas, entre outros) são concluídas, sendo que cada plugin pode ficar incubido de uma ou mais partes destes modelos. Sendo assim, existe bastante espaço para a criatividade na criação de novos plugins.

Como propostas de trabalho futuro, têm-se: (i) realizar uma extensão deste MSL, com o intuito de abordar as ferramentas utilizadas na literatura formal (artigos publicados em anais de conferência e periódicos científicos) e, então, analisar se elas podem ter alguma utilidade no contexto mercadológico; (ii) propor um novo plugin ou versão melhorada de um existente para o apoio à gamificação e disponibilizá-lo ao público acadêmico e do mercado de trabalho; e (iii) realizar um estudo de caso com professores que utilizam o Moodle para compreender melhor quais plugins eles utilizam, o porquê e quais elementos de gamificação não contemplados por plugins pré-existentes eles gostariam de usar.

\section{Referências}

Ortiz, S.; Green, M. Trends and patterns of mobile learning: A study of mobile learning management system access. Turkish Online Journal of Distance Education-TOJDE, v. 20, n. 1, p. 1, 2019.

Paulsen, M. F. Experiences with Learning Management Systems in 113 European Institutions. Educational Technology \& Society, v. 6, n. 4, p. 134, 2003.

Kapp, K. M. The gamification of learning and instruction: game-based methods and strategies for training and education. John Wiley \& Sons. 2012.

Kiryakova, G.; Angelova, N.; Yordanova, L. Gamification in education, 2014.

Dicheva, D.; Dichev, C.; Agre, G.; Angelova, G. Gamification in Education: A Systematic Mapping Study. Educational Technology \& Society, 2015.

Petersen, K.; Feldt, R.; Mujtaba, S.; Mattsson, M., Systematic Mapping Studies in Software Engineering, 2008.

Kitchenham, B.; Charters, S. Guidelines for performing systematic literature reviews in software engineering, Technical Report EBSE-2007-01, School of Computer Science and Mathematics, Keele University, 2007.

Lonn, S.; Teasley, S. D. Saving time or innovating practice: Investigating perceptions

and uses of Learning Management Systems Computers \& Education, 2009.

Petersen, K.; Feldt, R.; Mujtaba, S.; Mattsson M. Systematic mapping studies in software engineering Ease, 2008.

Moodle. Moodle Statistics, 2019. Disponível em: < $<$ https://moodle.net/stats/?lang=pt br $>$. Acesso em: 01 maio 2019. 